O. V.Zabolotnyi, Cand. Sc. (Tech.), Assoc. Prof., orcid.org/0000-0001-8266-4481,

V.A. Zabolotnyi, Cand. Sc. (Tech.), Assoc. Prof., orcid.org/0000-0001-9203-5080,

M. D. Koshevoi, Dr. Sc. (Tech.), Prof., orcid.org/0000-0001-9465-4467
State Higher Educational Institution "National Aerospace University "Kharkiv Aviation Institute", Kharkiv, Ukraine, e-mail: zzz77714@meta.ua; pretorian14@ukr.net; ndkoshevoy@ rambler.ru

\title{
CONDITIONALITY EXAMINATION OF THE NEW TESTING ALGORITHMS FOR COAL-WATER SLURRIES MOISTURE MEASUREMENT
}

\author{
О. В. Заболотний, канд. техн. наук, доц., \\ orcid.org/0000-0001-8266-4481, \\ В.А. Заболотний, канд. техн. наук, доц., \\ orcid.org/0000-0001-9203-5080, \\ М. Д. Кошовий, д-р техн. наук, проф., \\ orcid.org/0000-0001-9465-4467
}

\begin{abstract}
Державний вищий навчальний заклад „Національний аерокосмічний університет імені М. Є. Жуковського „Харківський авіаційний інститут“, м. Харків, Україна, е-таil: zzz77714@meta.ua; pretorian14@ukr.net; ndkoshevoy@ rambler.ru
\end{abstract}

\section{ПЕРЕВІРКА ЗУМОВЛЕНОСТІ НОВИХ ТЕСТОВИХ АЛГОРИТМІВ ДЛЯ ВИМІРЮВАННЯ ВМІСТУ ВОЛОГИ ВОДНО-ВУГІЛЬНИХ ЕМУЛЬСІЙ}

Purpose. To inspect the ability of the new testing algorithms of coal-water slurries moisture calculation to retain stability when working with experimental results that have natural random variation.

Methodology. Symmetric variation has been artificially introduced into the values of binary material-water slurry dielectric permittivity, applied in appropriate testing algorithm of moisture calculation. Inspection of the testing algorithm ability to retain the calculated water content values stability have been done for the conditions, when each value of dielectric permittivity that enters the testing algorithm takes maximum or minimum inside the symmetric variation range. Results of such an inspection allowed detecting some negative features of the existing testing algorithms and producing a new testing algorithm with sufficient stability for the calculated moisture values of material-water slurries.

Findings. When inspecting two testing algorithms for the calculated water content values conditionality, it was detected, in spite of our expectations, that variation of dielectric permittivity values in a rather small range of $0.1 \%$ gives a significant dispersion of calculated moister values. This situation signified low conditionality for both testing algorithms. It caused the authors to generate the modified testing algorithm, able to provide a sufficient stability for the calculated binary coal-water system moisture values according to the results of its comparison with modern analogues with a help of Pyrson's test.

Originality. Testing algorithms inspection for the calculated moisture values conditionality allowed generating more relevant testing algorithm of moisture calculating in binary systems and, as a result, increasing the accuracy of coal-water slurries moisture measurement.

Practical value. Application of the two additive, two multiplicative and two additional test influences on the substance under consideration in a new testing algorithm allowed increasing moisture measurement accuracy for capacitance moisture meters by several times. It is provided at the expense of small testing algorithm sensitivity to the substance's variation of physicochemical structure and due to the conditionality increase for the calculated values of moisture.

Keywords: moisture content control, capacitance moisture meter, dielectric permittivity, coal-water slurries

Introduction. Application of different coal slurries like COS (coal-oil slurry), CWS (coal-water slurry), COWS (coal-oil-water slurry), CMWS (coal-methanol-water slurry) is a way of further development for the coal-water suspension technologies [1,2].

The idea of CWS application instead of traditional oil products became widespread in the early $70 \mathrm{~s}$ of the $20^{\text {th }}$ century. Now the biggest range of CWS research studies can be seen in Japan and China [3]. As for Chinese meg-

(C) Zabolotnyi O. V., Zabolotnyi V.A., Koshevoi M. D., 2018 alopolises, it is forbidden to build and exploit boiler stations with solid coal. Besides, there is a state program to substitute traditional oil and gas fuel into CWS.

CWS usually contains $60-80 \%$ of specially grained coal, $20-40 \%$ of water and approximately $1 \%$ of chemical plasticizers, where moisture is an important parameter to control. Currently the capacitance method for moisture control prevails over others [4]. Its advantages are simple architecture, possibility of momentary measurements, satisfactory accuracy level and others. One of the main negative features of capacitance moisture me- 
ters is presence of the so-called "type uncertainty". It can be directly associated with a fact, that different dielectric materials under research have different dielectric permittivity values in a dehydrated stage.

Analysis of the recent research and publications. If we follow the reference [4], we can see that there are a lot of ways to solve the "type uncertainty" problem. Par example, such modern devices as Wile-55, Grain Master, Farmpoint, Multi Grain and Kett series, GAC500, are provided with calibrating tabular forms for the group of certain materials. As we claim to create a moisture meter for CWS moisture control to be universal for the wide range of coals, it is necessary to reduce the influence of "type uncertainty" as much as possible directly in a capacitance instrument transducer.

One of the perspective directions was detected for that purpose. The idea was to use special test methods that allow increasing the accuracy of measurements. Essentiality of these methods consists in determining the parameters of a static function for the transducer with the help of additional tests, functionally connected with an object under control. For the moisture control purposes test actions should be formed as a number of water injections into the substance under consideration. Using the values of dielectric permittivity after each of test actions it is possible to calculate the initial moisture of the CWS [5].

The authors explored various testing algorithms and detected a perspective combination, consisting of independent additive and multiplicative tests. Functional circuit of such a measuring system is illustrated below (Fig. 1).

According to Fig. 1, the measurement cycle consists of three steps. At the first step keys K1 and K2 are opened and key $\mathrm{K} 3$ is closed. Input parameter $\mathrm{X}$ is directly connected to the input of the meter M. At the second step keys K1 and $\mathrm{K} 3$ are closed, key K2 remains opened. Such a position for three keys forms an additive test action like $X+\Delta X$.

At the third step key K3 is opened and K2 is closed, which provides a multiplicative test $k \cdot X$.

Implementing such steps for our purposes, we can get a system of three equations (1)

$$
\left\{\begin{array}{l}
C_{1}=\varepsilon_{s}(1+3 W) \cdot g \\
C_{2}=\varepsilon_{s}(1+3(W+\Delta W)) \cdot g \\
C_{3}=k \cdot \varepsilon_{s}(1+3 W) \cdot g
\end{array}\right.
$$

where $\varepsilon_{s}$ is the dielectric permittivity of CWS; $g$ is the space characteristic of the electric field inside the capacitance instrument transducer gap between electrodes, $g=$ $10 \mathrm{~m} ; \Delta W$ is portion of water to get an additive test, $\Delta W=$ 0.1 ( $10 \%$ from the total volume of CWS); $k=2$ is the coefficient for the multiplicative test, $C_{1}, C_{2}, C_{3}$ are electric capacitances of the transducer.

After the system (1) solvation we can get an equation that allows moisture calculation (the first testing algorithm)

$$
W=\frac{\Delta W\left(C_{3}-C_{1}\right)}{(k-1)\left(C_{2}-C_{1}\right)}
$$

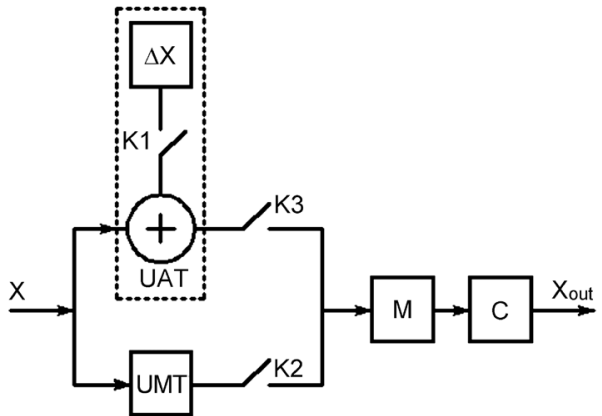

Fig. 1. Block diagram of a measuring system with additive and multiplicative tests and three keys:

$U A T$ - unit of additive test; UMT - unit of multiplicative test; $M$ - meter; $C$ - calculator; $K-$ key

In accordance with [5], after one more additive and multiplicative tests had been applied, a modified testing algorithm appeared

$$
\frac{W_{1}+W}{W_{1}-W}=\frac{\frac{\Delta W^{\prime}\left(C_{3}^{\prime}-C_{1}\right)}{\left(k^{\prime}-1\right)\left(C_{2}^{\prime}-C_{1}\right)}+\frac{\Delta W\left(C_{3}-C_{1}\right)}{(k-1)\left(C_{2}-C_{1}\right)}}{\frac{\Delta W^{\prime}\left(C_{3}^{\prime}-C_{1}\right)}{\left(k^{\prime}-1\right)\left(C_{2}^{\prime}-C_{1}\right)}-\frac{\Delta W\left(C_{3}-C_{1}\right)}{(k-1)\left(C_{2}-C_{1}\right)}},
$$

where $C_{2}^{\prime}=\varepsilon\left(1+3\left(W+\Delta W^{\prime}\right)\right) g ; C_{3}^{\prime}=k^{\prime} \varepsilon \times(1+3 W) g$; $\Delta W^{\prime}=0.2 ; k^{\prime}=4$ is the coefficient for the multiplicative test. Theoretical research studies on the test algorithm (2) show good results for the calculated moisture values $W$ when applying theoretical deterministic values $C_{1}, C_{2}, C_{3}, C_{2}^{\prime}, C_{3}^{\prime}$. But during the real CWS moisture control parameters of instrument transducer will not be deterministic and will have natural random variation. The task is to check how this fact will influence ability of the testing algorithm (2) to retain stability for the calculated values of moisture.

Objectives of the article. The main purpose of our research is to check the ability of the new testing algorithm for CWS moisture calculation to retain stability when working with experimental results that have natural random variation. A need for such a study is conditioned with a fact that values of electric capacitances taken from instrument transducer will not be ideal deterministic values used in [5] to check the testing algorithm effectiveness, but will be the results of measurements that always have some random variation. The level of random variation (capacitance measurement uncertainty) will directly influence the calculated moisture values conditionality: measurement uncertainty growth will decrease the calculated moisture values conditionality up to the moment when testing algorithm becomes irrelevant.

Scientific results. Symmetric variation, introduced into the values of binary material-water slurry dielectric permittivity, was calculated according to the universal Wiener equation [6]. Ranges of variation were set after metrological performance analysis for modern electric ca- 
pacitance meters had been carried out: $0.05,0.1,0.2$, $0.3 \%$ from the values of dielectric permittivity.
Calculated values of dielectric permittivity are shown in Tables 1-4 (initial values of dielectric permittivity

Table 1

Values of dielectric permittivity $\varepsilon_{s}$ with $0.05 \%$ variation for different moisture values $W$

\begin{tabular}{|c|c|c|c|c|c|c|c|c|c|c|c|c|}
\hline \multirow{2}{*}{$\begin{array}{l}W, \\
\%\end{array}$} & \multicolumn{12}{|c|}{$\varepsilon_{s}$} \\
\hline & 2. & 2 & $2+$ & 2.5 & 2.5 & $2.5_{+}$ & 3. & 3 & $3_{+}$ & 3.5 & 3.5 & $3.5_{+}$ \\
\hline 0 & 1.999 & 2.0 & 2.001 & 2.499 & 2.5 & 2.501 & 2.998 & 3.0 & 3.002 & 3.498 & 3.5 & 3.502 \\
\hline 10 & 2.613 & 2.614 & 2.615 & 3.250 & 3.252 & 3.254 & 3.883 & 3.885 & 3.887 & 4.510 & 4.512 & .514 \\
\hline 20 & 3.366 & 3.368 & 3.370 & 4.171 & 4.173 & 4.175 & 4.961 & 4.963 & 4.965 & 5.738 & 5.741 & 5.744 \\
\hline 30 & 4.315 & 4.317 & 4.319 & 5.321 & 5.324 & 5.327 & 6.302 & 6.305 & 6.308 & 7.258 & 7.262 & 7.266 \\
\hline 40 & 5.542 & 5.545 & 5.548 & 6.803 & 6.806 & 6.809 & 8.018 & 8.022 & 8.026 & 9.192 & 9.197 & 9.202 \\
\hline 50 & 7.196 & 7.2 & 7.204 & 8.780 & 8.784 & 8.788 & 10.290 & 10.295 & 10.300 & 11.732 & 11.738 & 11.744 \\
\hline 60 & 9.543 & 9.548 & 9.553 & 11.558 & 11.558 & 11.564 & 13.470 & 13.477 & 13.484 & 15.258 & 15.226 & 15.274 \\
\hline
\end{tabular}

Table 2

Values of dielectric permittivity $\varepsilon_{s}$ with $0.1 \%$ variation for different moisture values $W$

\begin{tabular}{|c|c|c|c|c|c|c|c|c|c|c|c|c|}
\hline \multirow{2}{*}{$\begin{array}{c}W \\
\%\end{array}$} & \multicolumn{10}{|c|}{$\varepsilon_{s}$} \\
\cline { 2 - 15 } & 2 & $\mathbf{2}$ & $2_{+}$ & 2.5 & $\mathbf{2 . 5}$ & $2.5_{+}$ & $3_{-}$ & $\mathbf{3}$ & $3_{+}$ & 3.5 & $\mathbf{3 . 5}$ & $3.5_{+}$ \\
\hline 0 & 1.998 & $\mathbf{2 . 0}$ & 2.002 & 2.498 & $\mathbf{2 . 5}$ & 2.502 & 2.997 & $\mathbf{3 . 0}$ & 3.003 & 3.497 & $\mathbf{3 . 5}$ & 3.503 \\
\hline 10 & 2.611 & $\mathbf{2 . 6 1 4}$ & 2.617 & 3.249 & $\mathbf{3 . 2 5 2}$ & 3.255 & 3.881 & $\mathbf{3 . 8 8 5}$ & 3.889 & 4.507 & $\mathbf{4 . 5 1 2}$ & 4.517 \\
\hline 20 & 3.365 & $\mathbf{3 . 3 6 8}$ & 3.371 & 4.169 & $\mathbf{4 . 1 7 3}$ & 4.177 & 4.958 & $\mathbf{4 . 9 6 3}$ & 4.968 & 5.735 & $\mathbf{5 . 7 4 1}$ & 5.747 \\
\hline 30 & 4.313 & $\mathbf{4 . 3 1 7}$ & 4.321 & 5.319 & $\mathbf{5 . 3 2 4}$ & 5.329 & 6.299 & $\mathbf{6 . 3 0 5}$ & 6.311 & 7.255 & $\mathbf{7 . 2 6 2}$ & 7.269 \\
\hline 40 & 5.539 & $\mathbf{5 . 5 4 5}$ & 5.551 & 6.799 & $\mathbf{6 . 8 0 6}$ & 6.813 & 8.014 & $\mathbf{8 . 0 2 2}$ & 8.030 & 9.188 & $\mathbf{9 . 1 9 7}$ & 9.206 \\
\hline 50 & 7.193 & $\mathbf{7 . 2}$ & 7.207 & 8.775 & $\mathbf{8 . 7 8 4}$ & 8.793 & 10.285 & $\mathbf{1 0 . 2 9 5}$ & 10.305 & 11.726 & $\mathbf{1 1 . 7 3 8}$ & 11.750 \\
\hline 60 & 9.538 & $\mathbf{9 . 5 4 8}$ & 9.558 & 11.546 & $\mathbf{1 1 . 5 5 8}$ & 11.570 & 13.464 & $\mathbf{1 3 . 4 7 7}$ & 13.490 & 15.251 & $\mathbf{1 5 . 2 2 6}$ & 15.281 \\
\hline
\end{tabular}

Table 3

Values of dielectric permittivity $\varepsilon_{s}$ with $0.2 \%$ variation for different moisture values $W$

\begin{tabular}{|c|c|c|c|c|c|c|c|c|c|c|c|c|}
\hline \multirow{2}{*}{$\begin{array}{c}W \\
\%\end{array}$} & \multicolumn{10}{|c|}{$\varepsilon_{s}$} \\
\cline { 2 - 15 } & 2 & 2 & $2_{+}$ & $2.5-$ & 2.5 & $2.5_{+}$ & $3_{-}$ & 3 & $3_{+}$ & $3.5-$ & 3.5 & $3.5_{+}$ \\
\hline 0 & 1.996 & 2.0 & 2.004 & 2.495 & $\mathbf{2 . 5}$ & 2.505 & 2.994 & $\mathbf{3 . 0}$ & 3.006 & 3.493 & $\mathbf{3 . 5}$ & 3.507 \\
\hline 10 & 2.609 & $\mathbf{2 . 6 1 4}$ & 2.619 & 3.245 & $\mathbf{3 . 2 5 2}$ & 3.259 & 3.877 & $\mathbf{3 . 8 8 5}$ & 3.893 & 4.503 & $\mathbf{4 . 5 1 2}$ & 4.521 \\
\hline 20 & 3.361 & $\mathbf{3 . 3 6 8}$ & 3.375 & 4.165 & $\mathbf{4 . 1 7 3}$ & 4.181 & 4.953 & $\mathbf{4 . 9 6 3}$ & 4.973 & 5.730 & $\mathbf{5 . 7 4 1}$ & 5.752 \\
\hline 30 & 4.308 & $\mathbf{4 . 3 1 7}$ & 4.326 & 5.313 & $\mathbf{5 . 3 2 4}$ & 5.335 & 6.292 & $\mathbf{6 . 3 0 5}$ & 6.318 & 7.247 & $\mathbf{7 . 2 6 2}$ & 7.277 \\
\hline 40 & 5.534 & $\mathbf{5 . 5 4 5}$ & 5.556 & 6.792 & $\mathbf{6 . 8 0 6}$ & 6.820 & 8.006 & $\mathbf{8 . 0 2 2}$ & 8.038 & 9.179 & $\mathbf{9 . 1 9 7}$ & 9.215 \\
\hline 50 & 7.186 & $\mathbf{7 . 2}$ & 7.214 & 8.766 & $\mathbf{8 . 7 8 4}$ & 8.802 & 10.274 & $\mathbf{1 0 . 2 9 5}$ & 10.316 & 11.715 & $\mathbf{1 1 . 7 3 8}$ & 11.761 \\
\hline 60 & 9.529 & $\mathbf{9 . 5 4 8}$ & 9.567 & 11.535 & $\mathbf{1 1 . 5 5 8}$ & 11.581 & 13.450 & $\mathbf{1 3 . 4 7 7}$ & 13.504 & 15.235 & $\mathbf{1 5 . 2 2 6}$ & 15.297 \\
\hline
\end{tabular}

Table 4

Values of dielectric permittivity $\varepsilon_{s}$ with $0.3 \%$ variation for different moisture values $W$

\begin{tabular}{|c|c|c|c|c|c|c|c|c|c|c|c|c|}
\hline \multirow{2}{*}{$\begin{array}{c}W \\
\%\end{array}$} & \multicolumn{10}{|c|}{$\varepsilon_{s}$} \\
\cline { 2 - 14 } & $2_{-}$ & $\mathbf{2}$ & $2_{+}$ & $2.5-$ & $\mathbf{2 . 5}$ & $2.5_{+}$ & $3_{-}$ & $\mathbf{3}$ & $3_{+}$ & $3.5-$ & $\mathbf{3 . 5}$ & $3.5_{+}$ \\
\hline 0 & 1.994 & $\mathbf{2 . 0}$ & 2.006 & 2.493 & $\mathbf{2 . 5}$ & 2.507 & 2.991 & $\mathbf{3 . 0}$ & 3.009 & 3.490 & $\mathbf{3 . 5}$ & 3.511 \\
\hline 10 & 2.606 & $\mathbf{2 . 6 1 4}$ & 2.622 & 3.242 & $\mathbf{3 . 2 5 2}$ & 3.262 & 3.873 & $\mathbf{3 . 8 8 5}$ & 3.897 & 4.498 & $\mathbf{4 . 5 1 2}$ & 4.526 \\
\hline 20 & 3.358 & $\mathbf{3 . 3 6 8}$ & 3.378 & 4.160 & $\mathbf{4 . 1 7 3}$ & 4.186 & 4.948 & $\mathbf{4 . 9 6 3}$ & 4.978 & 5.724 & $\mathbf{5 . 7 4 1}$ & 5.758 \\
\hline 30 & 4.304 & $\mathbf{4 . 3 1 7}$ & 4.330 & 5.308 & $\mathbf{5 . 3 2 4}$ & 5.340 & 6.286 & $\mathbf{6 . 3 0 5}$ & 6.324 & 7.240 & $\mathbf{7 . 2 6 2}$ & 7.284 \\
\hline 40 & 5.528 & $\mathbf{5 . 5 4 5}$ & 5.562 & 6.786 & $\mathbf{6 . 8 0 6}$ & 6.826 & 7.998 & $\mathbf{8 . 0 2 2}$ & 8.046 & 9.169 & $\mathbf{9 . 1 9 7}$ & 9.225 \\
\hline 50 & 7.178 & $\mathbf{7 . 2}$ & 7.222 & 8.758 & $\mathbf{8 . 7 8 4}$ & 8.810 & 10.264 & $\mathbf{1 0 . 2 9 5}$ & 10.326 & 11.703 & $\mathbf{1 1 . 7 3 8}$ & 11.773 \\
\hline 60 & 9.519 & $\mathbf{9 . 5 4 8}$ & 9.577 & 11.523 & $\mathbf{1 1 . 5 5 8}$ & 11.593 & 13.437 & $\mathbf{1 3 . 4 7 7}$ & 13.517 & 15.220 & $\mathbf{1 5 . 2 2 6}$ & 15.312 \\
\hline
\end{tabular}


calculated in accordance with universal Wiener equation are marked with bold print).

As mentioned before, inspection of the water content values conditionality was done for the conditions, when each value of dielectric permittivity that enters the testing algorithm takes maximum or minimum inside the symmetric variation range. Further the signs "+" and "-" will mean the maximum and minimal value, correspondingly, of dielectric permittivity $\varepsilon_{1}, \varepsilon_{2}$ or $\varepsilon_{3}$. As we have three parameters under control, eight different combinations will be possible (Table 5). Par example, designation like " ++" means that dielectric permittivities $\varepsilon_{1}$ and $\varepsilon_{3}$ take maximal values and $\varepsilon_{2}$ is minimal. Let us check testing algorithm (2) for the calculated moisture values stability.

As we can see from Table 5, testing algorithm (2) has low conditionality for moisture points 0,10 and $20 \%$ even with $0.05 \%$ variation. That is why the next attempt to create testing algorithm had been undertaken in accordance with a measuring system from Fig. 2.

Applying the block diagram in Fig. 2 we can get one more test $K(X+\Delta X)$ and system of equations (3)

$$
\left\{\begin{array}{l}
C_{1}=\varepsilon_{s}(1+3 W) \cdot g \\
C_{2}=\varepsilon_{s}(1+3(W+\Delta W)) \cdot g \\
C_{3}=k \cdot \varepsilon_{s}(1+3 W) \cdot g \\
C_{4}=k \cdot \varepsilon_{s}(1+3(W+\Delta W)) \cdot g
\end{array} .\right.
$$

In comparison with (1) this system will have one more equation. The solution of (3) gives us another equation for moisture calculation

$$
W=\frac{\left(C_{3}-C_{1}\right) \Delta W}{\left(C_{4}-C_{2}\right)-\left(C_{3}-C_{1}\right)}
$$

Acting in accordance with [5] after generating one more additive, multiplicative and complementary tests another modified testing algorithm appeared (4)

$$
W=\frac{\frac{\left(C_{3}^{\prime}-C_{1}\right) \Delta W^{\prime}}{\left(C_{4}^{\prime}-C_{2}^{\prime}\right)-\left(C_{3}^{\prime}-C_{1}\right)}+\frac{\left(C_{3}-C_{1}\right) \Delta W}{\left(C_{4}-C_{2}\right)-\left(C_{3}-C_{1}\right)}}{\left(C_{3}^{\prime}-C_{1}\right) \Delta W^{\prime}} .
$$

However, after conditionality inspection in a way illustrated in Table 5, it appeared that testing algorithm (4) and testing algorithm (2) have similar problems in moisture points 0 and $10 \%$.

The decision was to pay more attention to the testing algorithm (2) denominator after some modification (5)

$$
\begin{gathered}
W=\left[\left|\left(\frac{\Delta W^{\prime}\left(C_{3}^{\prime}-C_{1}\right)}{\left(k^{\prime}-1\right)\left(C_{2}^{\prime}-C_{1}\right)}-\frac{\Delta W\left(C_{3}-C_{1}\right)}{(k-1)\left(C_{2}-C_{1}\right)}\right)\right|-0.033\right] \times \\
\times 1600 .
\end{gathered}
$$

Inspection results for the testing algorithm (5) are placed in Table 6 . There we can see results of the $0.3 \%$ variation from the values of dielectric permittivity as most

Table 5

Moisture values $W$ with $0.05 \%$ variation according to (2)

\begin{tabular}{|c|c|c|c|c|c|c|c|c|c|}
\hline$\varepsilon_{s}$ & $W_{n}, \%$ & $W_{+++}$ & $W_{---}$ & $W_{-+}$ & $W_{+-+}$ & $W_{++-}$ & $W_{+--}$ & $W_{-++}$ & $W_{-+}$ \\
\hline 2.0 & 0 & 1.969 & 0.892 & -1.603 & 4.262 & -0.208 & 2.212 & 0.645 & 3.007 \\
\hline 2.0 & 10 & 11.068 & 11.905 & 8.793 & 13.997 & 9.688 & 12.732 & 10.206 & 13.199 \\
\hline 2.0 & 20 & 20.751 & 20.342 & 18.429 & 22.534 & 19.526 & 21.382 & 19.695 & 21.533 \\
\hline 2.0 & 30 & 30.448 & 30.518 & 28.948 & 31.935 & 29.563 & 31.102 & 29.852 & 31.369 \\
\hline 2.0 & 40 & 39.619 & 39.658 & 38.593 & 40.637 & 39.073 & 40.118 & 39.151 & 40.188 \\
\hline 2.5 & 0 & 0.881 & 1.966 & -2.136 & 4.706 & -0.966 & 3.034 & -0.239 & 3.681 \\
\hline 2.5 & 10 & 10.672 & 10.088 & 7.378 & 13.158 & 8.912 & 11.523 & 9.210 & 11.788 \\
\hline 2.5 & 20 & 20.360 & 20.791 & 18.388 & 22.623 & 19.315 & 21.656 & 19.463 & 21.784 \\
\hline 2.5 & 30 & 30.096 & 29.900 & 28.537 & 31.385 & 29.331 & 30.658 & 29.325 & 30.647 \\
\hline 2.5 & 40 & 39.463 & 39.683 & 38.767 & 40.351 & 39.178 & 40.079 & 39.057 & 39.960 \\
\hline 3.0 & 0 & 0.227 & 0.218 & -3.447 & 3.600 & -1.391 & 2.117 & -1.752 & 1.770 \\
\hline 3.0 & 10 & 10.512 & 10.001 & 7.652 & 12.691 & 8.977 & 11.252 & 9.240 & 11.489 \\
\hline 3.0 & 20 & 20.138 & 20.217 & 18.083 & 22.142 & 18.902 & 20.988 & 19.350 & 21.400 \\
\hline 3.0 & 30 & 29.662 & 29.709 & 28.082 & 31.199 & 28.795 & 30.385 & 28.971 & 30.544 \\
\hline 3.0 & 40 & 40.414 & 40.358 & 39.364 & 41.362 & 39.840 & 40.815 & 39.950 & 40.917 \\
\hline 3.5 & 0 & 0.355 & -0.361 & -3.641 & 3.348 & -1.806 & 1.348 & -1.388 & 1.722 \\
\hline 3.5 & 10 & 9.329 & 9.431 & 6.200 & 12.309 & 7.428 & 10.565 & 8.162 & 11.228 \\
\hline 3.5 & 20 & 19.838 & 19.904 & 17.299 & 22.251 & 18.425 & 20.949 & 18.761 & 21.249 \\
\hline 3.5 & 30 & 29.267 & 29.307 & 27.421 & 31.034 & 28.302 & 30.134 & 28.418 & 30.234 \\
\hline 3.5 & 40 & 40.444 & 40.389 & 39.291 & 41.486 & 39.838 & 40.912 & 39.912 & 40.977 \\
\hline
\end{tabular}




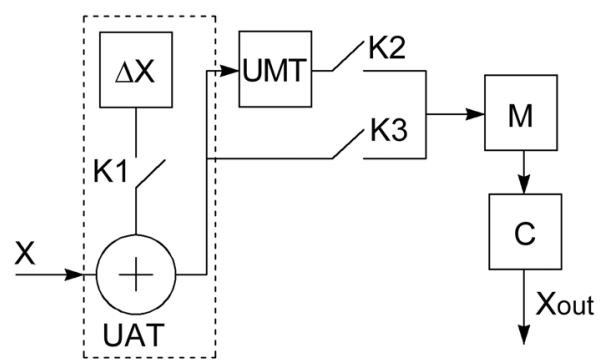

Fig. 2. Block diagram of a measuring system with additive, multiplicative and complementary tests

informative. Intermediate calculations for variation of 0.05 , 0.1 and $0.2 \%$ are absent in the article.

We can notice that bad conditionality of moisture results occurs for the combinations: $W_{-+-}, W_{++-}$and $W_{-++}$.
The next step of our research was to explore the modified denominator of the testing algorithm (4)

$$
\begin{gathered}
W=\mid \frac{\left(C_{3}^{\prime}-C_{1}\right) \Delta W^{\prime}}{\left(C_{4}^{\prime}-C_{2}^{\prime}\right)-\left(C_{3}^{\prime}-C_{1}\right)}-\frac{\left(C_{3}-C_{1}\right) \Delta W}{\left(C_{4}-C_{2}\right)-\left(C_{3}-C_{1}\right)}+ \\
+0.033 \mid \cdot 1666 .
\end{gathered}
$$

Results for the testing algorithm (6) can be found in Table 7 and again only variation of $0.3 \%$ is represented. Analyzing the results we can see that combinations $W_{-+-}$, $W_{++-}$and $W_{-++}$for the $0 \%$ moisture point give us moisture values, close to the testing algorithm (5) but with the opposite sign. This circumstance means that some average testing algorithm between $(5,6)$ would satisfy the purpose of the research and have good conditionality (7), Table 8.

$W=\left|\frac{\left(C_{3}^{\prime}-C_{1}\right) \Delta W^{\prime}}{\left(C_{4}^{\prime}-C_{2}^{\prime}\right)-\left(C_{3}^{\prime}-C_{1}\right)}-\frac{\left(C_{3}-C_{1}\right) \Delta W}{\left(C_{4}-C_{2}\right)-\left(C_{3}-C_{1}\right)}+0.033\right| \cdot 833+\left[\left|\frac{\Delta W^{\prime}\left(C_{3}^{\prime}-C_{1}\right)}{\left(k^{\prime}-1\right)\left(C_{2}^{\prime}-C_{1}\right)}-\frac{\Delta W\left(C_{3}-C_{1}\right)}{(k-1)\left(C_{2}-C_{1}\right)}\right|-0.033\right] \cdot 800$

Analyzing the data from Table 8, we can see that maximal conditionality decrease takes place for the $0.3 \%$ variation in a $40 \%$ moisture point and dielectric permittivity $\varepsilon_{s}=3.5$. However, if we compare these results with existing analogues, it is possible to make a conclusion that conditionality of moisture values, calculated with a help of testing algorithm (7), is satisfactory.

Experimental results. The first task was to create material-water samples with necessary percentage of water to provide test influences on the material under research. Air-oven reference method was used for that purpose [7]. To prepare our samples it was necessary to dry them up to the complete water extraction. Probes of different materials with a mass of $500 \mathrm{~g}$ were placed into gage tanks and weighed on the top-loading balance. After that gage tanks with opened lids were loaded into an oven, heated up to $120^{\circ} \mathrm{C}$, for 1.5 hours to dry samples inside gage tanks. For that purposes SNUG II-60 ana-

Table 6

Moisture values $W$ with $0.3 \%$ variation according to (5)

\begin{tabular}{|c|c|c|c|c|c|c|c|c|c|}
\hline$\varepsilon_{s}$ & $W_{n}, \%$ & $W_{+++}$ & $W_{--}$ & $W_{-+-}$ & $W_{+-+}$ & $W_{++-}$ & $W_{+--}$ & $W_{-++}$ & $W_{-++}$ \\
\hline 2.0 & 0 & 0.367 & 0.707 & -0.575 & 0.262 & -1.554 & 0.340 & -1.815 & 0.467 \\
\hline 2.0 & 10 & 10.879 & 10.549 & 8.185 & 11.959 & 9.286 & 11.365 & 9.591 & 10.956 \\
\hline 2.0 & 20 & 19.986 & 19.960 & 16.768 & 21.927 & 18.159 & 21.100 & 18.407 & 20.599 \\
\hline 2.0 & 30 & 30.423 & 30.594 & 27.388 & 32.382 & 29.021 & 31.980 & 28.614 & 30.820 \\
\hline 2.0 & 40 & 39.999 & 40.011 & 38.088 & 40.595 & 39.501 & 41.098 & 38.437 & 39.360 \\
\hline 2.5 & 0 & 0.677 & 1.190 & -0.661 & 1.133 & -0.839 & 0.618 & -1.311 & 1.539 \\
\hline 2.5 & 10 & 9.719 & 10.105 & 7.458 & 12.074 & 7.863 & 11.217 & 8.202 & 10.764 \\
\hline 2.5 & 20 & 20.187 & 20.060 & 16.337 & 22.740 & 18.381 & 21.934 & 17.943 & 20.666 \\
\hline 2.5 & 30 & 29.717 & 29.478 & 26.337 & 31.622 & 28.225 & 31.130 & 27.647 & 29.789 \\
\hline 2.5 & 40 & 39.467 & 39.372 & 37.226 & 40.283 & 38.638 & 40.454 & 37.901 & 39.047 \\
\hline 3.0 & 0 & 0.342 & 0.710 & -1.663 & 1.402 & -1.224 & 0.836 & -0.879 & 1.094 \\
\hline 3.0 & 10 & 10.180 & 10.029 & 7.622 & 12.644 & 8.007 & 11.471 & 8.342 & 10.992 \\
\hline 3.0 & 20 & 19.721 & 19.755 & 15.414 & 22.912 & 17.468 & 21.659 & 17.458 & 20.798 \\
\hline 3.0 & 30 & 29.129 & 29.015 & 24.993 & 31.977 & 27.171 & 31.020 & 26.754 & 29.776 \\
\hline 3.0 & 40 & 41.219 & 41.374 & 38.334 & 43.002 & 40.236 & 43.019 & 39.155 & 41.195 \\
\hline 3.5 & 0 & 0.649 & 1.258 & -1.714 & 2.350 & -1.033 & 1.668 & -0.220 & 1.752 \\
\hline 3.5 & 10 & 9.858 & 9.284 & 6.556 & 12.540 & 7.344 & 11.026 & 7.734 & 10.574 \\
\hline 3.5 & 20 & 19.466 & 19.624 & 23.664 & 23.390 & 16.740 & 21.665 & 17.089 & 21.128 \\
\hline 3.5 & 30 & 28.390 & 28.606 & 31.680 & 32.228 & 26.165 & 31.003 & 25.680 & 29.622 \\
\hline 3.5 & 40 & 41.510 & 41.251 & 44.950 & 43.874 & 40.068 & 43.431 & 38.950 & 41.513 \\
\hline
\end{tabular}


Moisture values $W$ with $0.3 \%$ variation according to (6)

\begin{tabular}{|c|c|c|c|c|c|c|c|c|c|}
\hline$\varepsilon_{s}$ & $W_{n}, \%$ & $W_{+++}$ & $W_{--}$ & $W_{-+-}$ & $W_{+-+}$ & $W_{++-}$ & $W_{+--}$ & $W_{-++}$ & $W_{-+}$ \\
\hline 2.0 & 0 & 0.382 & 0.736 & 0.973 & 0.262 & 1.824 & 0.340 & 0.855 & 1.467 \\
\hline 2.0 & 10 & 11.328 & 10.984 & 12.094 & 11.959 & 8.421 & 12.365 & 10.739 & 12.956 \\
\hline 2.0 & 20 & 20.810 & 20.784 & 20.965 & 21.927 & 18.661 & 22.100 & 19.919 & 22.599 \\
\hline 2.0 & 30 & 31.678 & 31.856 & 32.023 & 32.382 & 28.970 & 32.980 & 30.547 & 32.820 \\
\hline 2.0 & 40 & 41.649 & 41.662 & 43.164 & 40.595 & 39.883 & 42.098 & 40.775 & 41.360 \\
\hline 2.5 & 0 & 0.704 & 1.239 & 1.183 & 1.758 & 2.121 & 0.932 & 0.130 & 2.891 \\
\hline 2.5 & 10 & 10.120 & 10.522 & 12.683 & 12.074 & 7.940 & 12.968 & 9.292 & 13.497 \\
\hline 2.5 & 20 & 21.020 & 20.888 & 21.516 & 22.740 & 17.892 & 21.128 & 19.436 & 23.807 \\
\hline 2.5 & 30 & 30.943 & 30.694 & 32.228 & 33.504 & 28.142 & 33.703 & 29.540 & 33.306 \\
\hline 2.5 & 40 & 41.092 & 40.996 & 43.267 & 40.283 & 38.984 & 42.412 & 40.217 & 42.946 \\
\hline 3.0 & 0 & 0.356 & 0.739 & 1.827 & 1.858 & 2.522 & 1.160 & 0.994 & 1.428 \\
\hline 3.0 & 10 & 10.600 & 10.442 & 12.853 & 13.743 & 7.090 & 13.233 & 9.438 & 12.734 \\
\hline 3.0 & 20 & 20.534 & 20.570 & 21.655 & 22.935 & 16.941 & 21.842 & 18.930 & 23.045 \\
\hline 3.0 & 30 & 30.330 & 30.212 & 33.529 & 33.874 & 28.045 & 33.988 & 28.610 & 32.293 \\
\hline$\varepsilon_{s}$ & $W_{n}, \%$ & $W_{+++}$ & $W_{--}$ & $W_{-+-}$ & $W_{+-+}$ & $W_{++-}$ & $W_{+--}$ & $W_{-++}$ & $W_{-+}$ \\
\hline 3.0 & 40 & 42.920 & 43.080 & 43.920 & 42.354 & 40.648 & 43.082 & 40.522 & 44.183 \\
\hline 3.5 & 0 & 0.676 & 1.310 & 2.280 & 2.025 & 3.323 & 1.426 & 0.634 & 1.113 \\
\hline 3.5 & 10 & 10.265 & 9.667 & 12.827 & 12.635 & 7.400 & 13.770 & 8.806 & 13.299 \\
\hline 3.5 & 20 & 20.269 & 20.433 & 22.691 & 23.132 & 17.183 & 22.247 & 18.547 & 23.289 \\
\hline 3.5 & 30 & 29.561 & 29.786 & 32.745 & 34.135 & 27.997 & 34.170 & 28.492 & 33.133 \\
\hline 3.5 & 40 & 42.842 & 42.952 & 44.747 & 43.261 & 38.473 & 43.512 & 39.309 & 44.514 \\
\hline
\end{tabular}

Table 8

Moisture values $W$ with $0.3 \%$ variation according to (7)

\begin{tabular}{|c|c|c|c|c|c|c|c|c|c|}
\hline$\varepsilon_{s}$ & $W_{n}, \%$ & $W_{+++}$ & $W_{--}$ & $W_{-+-}$ & $W_{+-+}$ & $W_{++-}$ & $W_{+--}$ & $W_{-++}$ & $W_{-+}$ \\
\hline 2.0 & 0 & 0.375 & 0.713 & 0.199 & 0.556 & 0.135 & 0.492 & -0.480 & 1.121 \\
\hline 2.0 & 10 & 11.104 & 10.767 & 10.140 & 12.495 & 8.854 & 11.744 & 10.165 & 11.326 \\
\hline 2.0 & 20 & 20.398 & 20.372 & 18.867 & 22.668 & 20.294 & 21.680 & 19.163 & 21.169 \\
\hline 2.0 & 30 & 31.051 & 31.225 & 29.706 & 32.839 & 28.996 & 32.284 & 29.581 & 31.600 \\
\hline 2.0 & 40 & 40.280 & 40.837 & 40.626 & 41.720 & 39.692 & 42.090 & 39.606 & 40.317 \\
\hline 2.5 & 0 & 0.691 & 1.215 & 0.261 & 1.446 & 0.641 & 0.775 & -0.591 & 2.225 \\
\hline 2.5 & 10 & 9.920 & 10.314 & 10.071 & 12.612 & 7.902 & 12.093 & 8.747 & 12.131 \\
\hline 2.5 & 20 & 20.694 & 20.474 & 18.927 & 23.498 & 18.367 & 21.531 & 18.465 & 22.237 \\
\hline 2.5 & 30 & 30.330 & 30.086 & 29.283 & 32.563 & 28.184 & 32.417 & 28.594 & 31.548 \\
\hline 2.5 & 40 & 40.280 & 40.184 & 40.247 & 41.403 & 38.811 & 41.433 & 39.059 & 40.997 \\
\hline 3.0 & 0 & 0.349 & 0.725 & 0.082 & 1.630 & 0.649 & 0.998 & 0.058 & 1.261 \\
\hline 3.0 & 10 & 10.390 & 10.236 & 10.238 & 13.194 & 7.549 & 12.352 & 8.845 & 11.863 \\
\hline 3.0 & 20 & 20.128 & 20.163 & 18.535 & 22.924 & 17.205 & 21.751 & 18.194 & 21.922 \\
\hline 3.0 & 30 & 29.730 & 29.614 & 29.261 & 32.926 & 27.608 & 32.504 & 27.682 & 31.035 \\
\hline 3.0 & 40 & 42.070 & 42.227 & 41.127 & 42.678 & 40.442 & 43.051 & 39.854 & 42.689 \\
\hline 3.5 & 0 & 0.663 & 1.284 & 0.283 & 2.188 & 1.145 & 1.547 & 0.217 & 1.433 \\
\hline 3.5 & 10 & 10.062 & 9.476 & 9.692 & 12.588 & 7.372 & 12.398 & 8.270 & 11.937 \\
\hline 3.5 & 20 & 19.868 & 20.029 & 23.178 & 23.261 & 16.962 & 21.956 & 17.817 & 22.209 \\
\hline 3.5 & 30 & 28.976 & 29.196 & 32.213 & 33.182 & 27.081 & 32.587 & 27.086 & 31.378 \\
\hline 3.5 & 40 & 42.176 & 42.102 & 44.849 & 43.568 & 39.271 & 43.472 & 39.130 & 43.014 \\
\hline
\end{tabular}


lytic top-loading balance and TBV-2000 thermal vacuum chamber were used.

After being heated gage tanks were covered with lids, cooled down to $+20{ }^{\circ} \mathrm{C}$ temperature and weighed with $\pm 0.01 \mathrm{~g}$ accuracy. Cycles of drying should be continued till the mass of gage tank become stable. That will provide complete moisture deletion.

Samples taken for the research were represented with wheat, barley, pearl barley, poppy-seed, crushed pea and sand - dry materials with rather different values of dielectric permittivity and grain-size composition.

It should be clear that each of six probes in dehydrated state is a sample with $0 \%$ of moisture. Next sample with $10 \%$ of moisture can be created by adding $50 \mathrm{~g}$ of water in accordance with [7]. Third sample - $100 \mathrm{~g}$ $(20 \%)$, fourth $-150 \mathrm{~g}(30 \%)$, etc.

Two capacitance instrument transducers T1 and T2 with different plates shape were used to measure dielectric permittivity of the prepared samples (Fig. 3).

T1 instrument transducer consists of two parallel teflon-covered plates, assembled on a polytetra-fluorethylene disk with a capacitance in empty state equal to $7 \mathrm{pF}$. Top of T1 transducer has two clamps to connect the capacitance meter. T2 instrument transducer contains a system of flat plates that form nine electric capacitances connected in parallel and coated with epoxy varnish. Electric capacitance of $\mathrm{T} 2$ instrument transducer in empty state is equal to $47 \mathrm{pF}$. System of flat plates is assembled inside two polytetra-fluorethylene rings with tang slots. Dielectric coat of both instrument transducers is necessary to prevent the electrical short-circuit of electrodes when the sample under research moisture value overcomes $10 \%$. Two instrument transducers application allowed two groups of independent results to be obtained with an ability to be compared.

Unfortunately, all list of RLC and capacitance meters, portable and bench multimeter instruments as well as three types of Q-meters we had at our disposal failed to measure electric capacitances of both instrument transducers correctly because of strong dielectric losses in moisture samples. That is why original capacitance - pulse duration - voltage secondary transducer had been developed (Fig. 4).

Mentioned above capacitance-voltage transducer is based on two 555 or 777 timers (Fig. 5), where the left one is connected as a multivibrator circuit and generates meander pulses on its output. The right one, where instrument transducer with moisture sample $\mathrm{C}_{\mathrm{x} 1}$ is connected, works as a one-shot multivibrator, and pulses duration on its output is in straight proportion with a capacitance of the instrument transducer. Low-pass filter $\mathrm{R}_{4} \mathrm{C}_{4}$ works as pulse duration into d. c. voltage transducer in a range of $0.000-2.000$ volts.

Measurement process included several steps. At the first step instrument transducer filled with a prepared sample were connected to the secondary transducer. Oscilloscope was used to control the form and duration of output pulses and the task of a digital multimeter was to measure output d. c. voltage of the circuit. D. c. voltage value was fixed by the operator. At the second step instrument transducer with a sample was disconnected form the cir-

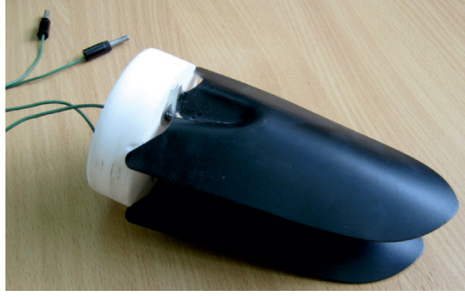

$a$

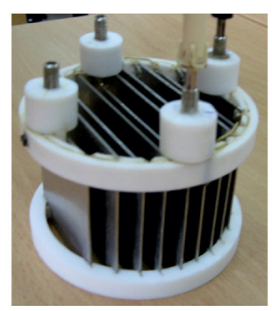

$b$
Fig. 3. Capacitance instrument transducers:

$a-T 1$ with two teflon-coated plates; $b-T 2$ with multiple epoxy-varnished plates

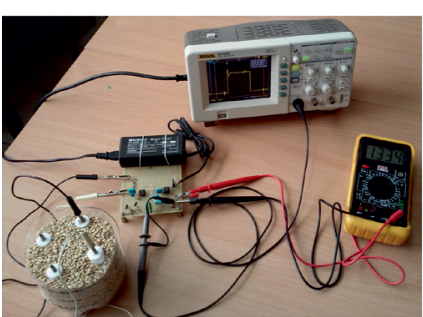

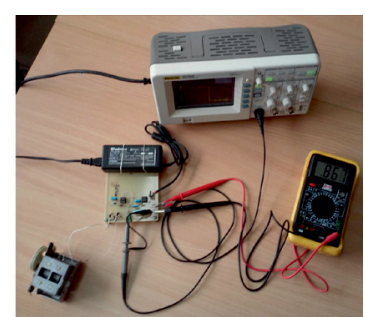

$b$
Fig. 4. Laboratory setup for capacitance measurement: $a$ - with moisture sample; $b$ - with variable air capacitor instead moisture sample

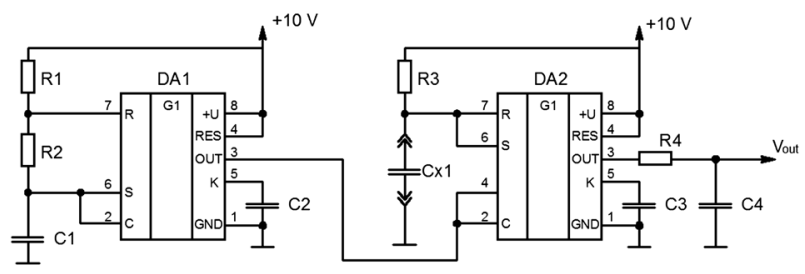

Fig. 5. Electric circuit of a capacitance-voltage transducer

cuit and substituted with variable air capacitor (Fig. 4, b) able to change its capacitance in a range of 5-760 $\mathrm{pF}$. Capacitance of the air capacitor in accordance with a substitution method of measurement was slowly increased till the output voltage value was equal to the value, fixed by the operator. Then air capacitor was disconnected from the circuit and its capacitance was measured with high accuracy.

Average values for ten results of capacitance measurement are shown in Tables 9, 10 (for both instrument transducers).

Such a number of samples were necessary to cover the moisture range from 0 up to $30 \%$ what is enough for CWS moisture control.

Substituting these results into equation (7) we were able to get experimental values of moisture, placed in Tables 11 and 12 .

Conclusion. As we can see, experimental results of moisture measurement are close to the nominal moisture points, which means that testing algorithm (7) can be applied with experimental results of measurement and retains a good conditionality of calculated moisture values. Besides, we can see that testing algorithm (7) demonstrates good results in solving the "type uncertainty" 
Table 9 problem because moister values of different materials are

Values of capacitance for T1 instrument transducer

\begin{tabular}{|l|c|c|c|c|c|c|}
\hline & \multicolumn{6}{|c|}{$C, \mathrm{pF}$} \\
\hline \multicolumn{1}{|c|}{$W, \%$} & 0 & 10 & 20 & 30 & 40 & 50 \\
\hline Wheat & 28.0 & 36.2 & 45.7 & 57.7 & 72.3 & 91.8 \\
\hline Barley & 22.4 & 29.2 & 37.1 & 47.2 & 59.5 & 76.2 \\
\hline $\begin{array}{l}\text { Pearl } \\
\text { barley }\end{array}$ & 26.4 & 34.5 & 43.9 & 55.8 & 68.7 & 87.4 \\
\hline $\begin{array}{l}\text { Poppy- } \\
\text { seed }\end{array}$ & 25.2 & 32.7 & 41.4 & 52.5 & 66.0 & 84.1 \\
\hline $\begin{array}{l}\text { Crushed } \\
\text { pea }\end{array}$ & 23.1 & 30.1 & 38.2 & 48.5 & 61.1 & 78.2 \\
\hline Sand & 14.0 & 18.3 & 23.3 & 29.8 & 38.8 & 50.4 \\
\hline
\end{tabular}

Table 10

Values of capacitance for T2 instrument transducer

\begin{tabular}{|l|c|c|c|c|c|c|}
\hline & \multicolumn{6}{|c|}{$C, \mathrm{pF}$} \\
\hline \multicolumn{1}{|c|}{$W, \%$} & 0 & 10 & 20 & 30 & 40 & 50 \\
\hline Wheat & 188.1 & 241.7 & 306.2 & 386.0 & 485.7 & 616.6 \\
\hline Barley & 150.3 & 194.3 & 248.2 & 316.5 & 399.1 & 511.1 \\
\hline $\begin{array}{l}\text { Pearl } \\
\text { barley }\end{array}$ & 178.5 & 229.8 & 291.7 & 368.7 & 464.3 & 590.7 \\
\hline $\begin{array}{l}\text { Poppy- } \\
\text { seed }\end{array}$ & 169.1 & 218.0 & 277.1 & 351.0 & 442.9 & 564.7 \\
\hline $\begin{array}{l}\text { Crushed } \\
\text { pea }\end{array}$ & 155.1 & 200.3 & 255.3 & 324.5 & 410.2 & 524.7 \\
\hline Sand & 94.1 & 122.5 & 158.5 & 205.9 & 260.6 & 338.4 \\
\hline
\end{tabular}

Table 11

Experimental values of moisture for $\mathrm{T} 1$ transducer

\begin{tabular}{|l|c|c|c|c|}
\hline \multicolumn{1}{|c|}{$W, \%$} & 0 & 10 & 20 & 30 \\
\hline Wheat & 0.388 & 9.763 & 19.921 & 30.041 \\
\hline Barley & 0.419 & 9.481 & 19.802 & 29.738 \\
\hline $\begin{array}{l}\text { Pearl } \\
\text { barley }\end{array}$ & 0.401 & 9.605 & 19.731 & 29.701 \\
\hline $\begin{array}{l}\text { Poppy- } \\
\text { seed }\end{array}$ & 0.237 & 10.000 & 20.114 & 30.254 \\
\hline $\begin{array}{l}\text { Crushed } \\
\text { pea }\end{array}$ & 0.084 & 9.835 & 19.967 & 30.126 \\
\hline Sand & 0.000 & 9.786 & 19.872 & 29.917 \\
\hline
\end{tabular}

Table 12

Experimental values of moisture for $\mathrm{T} 2$ transducer

\begin{tabular}{|l|c|c|c|c|}
\hline \multicolumn{1}{|c|}{$W, \%$} & 0 & 10 & 20 & 30 \\
\hline Wheat & 0.397 & 9.881 & 19.885 & 29.844 \\
\hline Barley & 0.472 & 10.000 & 20.351 & 30.436 \\
\hline $\begin{array}{l}\text { Pearl } \\
\text { barley }\end{array}$ & 0.272 & 9.893 & 20.191 & 30.210 \\
\hline $\begin{array}{l}\text { Poppy- } \\
\text { seed }\end{array}$ & 0.000 & 9.842 & 19.793 & 29.710 \\
\hline $\begin{array}{l}\text { Crushed } \\
\text { pea }\end{array}$ & 0.011 & 9.679 & 19.699 & 29.672 \\
\hline Sand & 0.366 & 9.594 & 19.701 & 29.843 \\
\hline
\end{tabular}

close to each other.

Testing algorithms inspection for the calculated moisture values conditionality allowed generating more relevant testing algorithm of moisture calculating in binary systems and, as a result, increasing the accuracy of coalwater slurries moisture measurement.

\section{References.}

1. Arashdeep Singh, Puneet Juneja, Umesh, Satish Kumar and S. K. Mohapatra, 2015. Influence of Particle Size and Temperature on Rheological Characteristics of Coal Oil Suspension. International Journal of Engineering Technology, Management and Applied Sciences, 3, pp. 44-50.

2. Ugwu, K.E. and Eze, S. I., 2014. Physicochemical and rheological characteristics of charcoal slurry fuel. International Journal of Energy and Environment (IJEE), 5(1), pp. 119-126.

3. Jian Xu, Yong Yang and Yong-Wang Li, 2015. Recent Development in Converting Coal to Clean Fuels in China. Fuel, 152, pp. 122-130.

4. Nelson, S. O. and Trabelsi, S., 2014. A century of grain and seed moisture measurement by sensing electrical properties. Transactions of the ASABE, 55(2), pp. 629636.

5. Holub, K. Yu., Zabolotnyi, O.V. and Koshevoi, M.D., 2014. Synthesis of Test Actions for Capacitive Moisture Meter that is Invariant to Substance Type Change, TEKA. Commission of motorization and energetics in agriculture, 14(2), pp. 43-52.

6. Josh, M. and Clennell, B., 2015. Broadband electrical properties of clays and shales: Comparative investigations of remolded and preserved samples, Geophysics, 80(2), pp. 129-143.

7. G. Diane Lee and Harris, G., 2016. Examination of Grain Moisture Meters Using Air-Oven Reference Method Transfer Standards. - Natl. Inst. Stand. Technol., 2016 Ed., 78 p.

Мета. Перевірка здатності нових тестових алгоритмів розрахунку вмісту вологи водно-вугільних емульсій зберігати зумовленість обчислених рішень під час роботи з експериментальними даними, що мають природну варіацію.

Методика. У значення діелектричних проникностей бінарної суміші матеріал - вода, за якими здійснюється розрахунок вмісту вологи за обраним тестовим алгоритмом, штучно вводиться симетрична варіація. Здійснена перевірка здатності тестового алгоритму зберігати стабільність розрахункових значень вмісту вологи в умовах, коли кожна з діелектричних проникностей бінарної суміші, що входять у тестовий алгоритм, приймає максимальне або мінімальне значення в межах заданої варіації. Результати перевірки дозволили виявити недоліки запропонованих раніше тестових алгоритмів і синтезувати новий, що має достатній рівень зумовленості розрахункових значень вмісту вологи.

Результати. Унаслідок перевірки на зумовленість двох розглянутих тестових алгоритмів виявилось, що, 
всупереч очікуванням, уже за наявності варіації значень діелектричних проникностей на рівні $0,1 \%$ спостерігається значне розсіювання розрахункових значень вмісту вологи, що свідчить про погану зумовленість отриманих рішень. Це викликало необхідність синтезу ще одного тестового алгоритму, який забезпечує задовільну зумовленість розрахункових значень вмісту вологи в результаті його порівняння з аналогами за допомогою критерію узгодженості Пірсона.

Наукова новизна. Перевірка відомих тестових алгоритмів на зумовленість отриманих рішень дозволила синтезувати більш досконалий тестовий алгоритм розрахунку вмісту вологи бінарних систем, i, як наслідок, підвищити точність вимірювання вмісту вологи водно-вугільних емульсій.

Практична значимість. Використання двох адитивних, Двох мультиплікативних і двох додаткових тестових впливів на досліджувану речовину в новому тестовому алгоритмі дозволило в декілька разів підвищити точність вимірювання вмісту вологи ємнісними вологомірами. Це вдалося забезпечити як за рахунок низької чутливості тестового алгоритму до зміни фізико-хімічного складу досліджуваної речовини, так і за рахунок підвищення зумовленості розрахункових значень вмісту вологи.

Ключові слова: контроль вмісту вологи, ємнісний вимірювач вологості, діелектрична проникність, водно-вугільна емульсія

Цель. Проверка способности новых тестовых алгоритмов расчета влагосодержания водно-угольных эмульсий сохранять обусловленность получаемых решений при работе с экспериментальными данными, имеющими природную вариацию.

Методика. В значения диэлектрических проницаемостей бинарной смеси материал - вода, на основании которых производится расчет влагосодержания по выбранному тестовому алгоритму, искусственно вводится симметричная вариация. Осуществлена проверка способности тестового алгоритма сохранять стабильность расчетных значений влагосодержания в условиях, когда каждая из диэлектриче- ских проницаемостей бинарной смеси, входящих в тестовый алгоритм, принимает максимальное или минимальное значение в пределах заданной вариации. Результаты проверки позволили выявить недостатки предложенных ранее тестовых алгоритмов и синтезировать новый, имеющий достаточную степень обусловленности расчетных значений влагосодержания.

Результаты. В результате проверки на обусловленность двух рассмотренных тестовых алгоритмов оказалось, что, вопреки ожиданиям, уже при вариации значений диэлектрических проницаемостей на уровне $0,1 \%$ наблюдается значительный разброс расчетных значений влагосодержания, что говорит о плохой обусловленности получаемых решений. Это вызвало необходимость синтезировать еще один тестовый алгоритм, обеспечивающий удовлетворительную обусловленность расчетных значений влагосодержания по результатам его сравнения с аналогами при помощи критерия согласия Пирсона.

Научная новизна. Проверка известных тестовых алгоритмов на обусловленность получаемых решений позволила синтезировать более совершенный тестовый алгоритм расчета влагосодержания бинарных систем, и, как следствие, повысить точность измерения влагосодержания водно-угольных эмульсий.

Практическая значимость. Применение двух аддитивных, двух мультипликативных и двух дополнительных тестовых воздействий на исследуемое вещество в новом тестовом алгоритме позволило в несколько раз повысить точность измерения влагосодержания емкостными влагомерами. Это обеспечивается как за счет слабой чувствительности тестового алгоритма к изменению физико-химического состава исследуемого вещества, так и за счет повышения обусловленности расчетных значений влагосодержания.

Ключевые слова: контроль влагосодержания, емкостный измеритель влажности, диэлектрическая проницаемость, водно-угольная эмульсия

Рекомендовано до публікації докт. техн. наук С.I. Кондрашовим. Датанадходження рукопису 07.12.16. 